Case Report

\title{
Coexistence of Neonatal Lupus Erythematous and Sturge-Weber Syndrome
}

\author{
Zahra Nikyar (D), 1,2 Parvaneh Hatami $\left(\mathbb{D},{ }^{1}\right.$ Zeinab Aryanian (iD, ${ }^{1,3}$ Kambiz Kamyab Hesari $\left(\mathbb{D},{ }^{4}\right.$ \\ Azadeh Goodarzi $\mathbb{D}^{5},{ }^{5}$ and Anahita Borzouei ${ }^{6}$ \\ ${ }^{1}$ Autoimmune Bullous Diseases Research Center, Tehran University of Medical Sciences, Tehran, Iran \\ ${ }^{2}$ Department of Dermatology, School of Medicine, Razi Hospital, Tehran University of Medical Sciences, Tehran, Iran \\ ${ }^{3}$ Department of Dermatology, Babol University of Medical Sciences, Babol, Iran \\ ${ }^{4}$ Department of Dermatopathology, School of Medicine Razi Hospital, Tehran University of Medical Sciences, Tehran, Iran \\ ${ }^{5}$ Department of Dermatology, School of Medicine, Rasoul Akram Hospital, Iran University of Medical Sciences, Tehran, Iran \\ ${ }^{6}$ Department of Dermatology, Mashhad University of Medical Sciences, Mashhad, Iran
}

Correspondence should be addressed to Parvaneh Hatami; p_hatami2001@yahoo.com and Zeinab Aryanian; z_aryanian@ yahoo.com

Received 5 September 2021; Revised 4 December 2021; Accepted 17 December 2021; Published 30 December 2021

Academic Editor: Hristo Dobrev

Copyright (C) 2021 Zahra Nikyar et al. This is an open access article distributed under the Creative Commons Attribution License, which permits unrestricted use, distribution, and reproduction in any medium, provided the original work is properly cited.

Neonatal lupus erythematous (NLE) is a rare condition presented by lupus dermatitis shortly after birth or later following sun exposure. Sturge-Weber syndrome (SWS) is also an uncommon congenital condition characterized by extensive capillary malformation and ophthalmic and/or neurologic involvement. Here, we describe the first case of coexistence of NLE and SWS which posed a significant diagnostic challenge to clinicians.

\section{Introduction}

Neonatal lupus erythematous (NLE) is a rare condition produced by the transplacental passage of maternal autoantibodies [1]. NLE usually presented with lupus dermatitis including annular erythemato-squamous plaques developing shortly after birth or later following sun exposure [2].

The skin lesions often resolve spontaneously with or without leaving hypopigmented or hyperpigmented scar within the first year of life.

Sturge-Weber syndrome (SWS) is also an uncommon congenital condition characterized by extensive capillary malformation and ophthalmic and/or neurologic involvement [3].

Here, we describe a case that looks worthy of record since it presented with both NLE and SWS which has not been previously reported.

\section{Case Report}

A 3-month-old girl, born to nonconsanguineous parents, presented to our clinic with extensive port-wine stain on the left facial area since birth (Figure 1).

She was the full-term production of an uncomplicated pregnancy to a healthy mother without a history of abortion or any known medical condition.

On physical examination, the patient had a generally good condition. An extensive port-wine stain was noted distributing on V1, V2, and V3 areas on the left side of the face without involvement of mucosa. Multiple atrophic and hyperpigmented patches with telangiectasia on both sides of the face were seen which were more prominent on the forehead and temporal region and around the eyes. They presented since birth and were more obvious on the right side of the face due to presence of the port-wine stain on the 
other side. Multiple erythematous and/or hyperpigmented punched-out lesions, some with scaly appearance and/or atrophic surface, were noted on the trunk and proximal parts of extremities (Figure 2).

Initially, it was assumed to be a case of SWS and the patient was referred to the pediatric department for further ophthalmologic and neurologic examinations regarding presence of any abnormality in favor of SWS. No remarkable finding was noted in evaluations, except for bilateral benign horn cyst found in brain sonography.

However, we did not have any explanation for her atrophic punched-out lesions in the setting of SWS. Therefore, histiocytosis, mastocytosis, Goltz syndrome, and scar formation following maternal viral or parasitic infections during pregnancy including TORCH infections (toxoplasmosis, rubella, cytomegalovirus, and herpes simplex) were considered as the most relevant differential diagnoses, despite the absence of any applicable maternal history. A $4 \mathrm{~mm}$ punch biopsy was taken from one of the atrophic lesions of her trunk. Histopathologic assessment showed dermal infiltration of spindle cells in a collagenous stroma containing abundant mucin intermixed with some mast cells which was more compatible with "progressive mucinous histiocytosis" (Figures 3 and 4). "Cutaneous mucinosis of infancy" and "self-healing juvenile cutaneous mucinosis" were also suggested as differential diagnoses.

However, clinical manifestation of these disorders was quite different from that seen in our case.

Moreover, IHC study for CD 31, S100, C-kit, and lysozyme was performed which was not diagnostic for any of these diagnoses.

Unfortunately, we could not perform serologic evaluation for TORCH infections because of some financial issues.

Considering clinical appearance of the lesions including atrophy and telangiectasia and existence of abundant mucin in histopathologic assessment which has been reported as a rare finding in patients with lupus erythematosus [4], a diagnosis of NLE was suspected and relevant laboratory data were requested. Serologic examination of sera of the patient and her mother revealed the presence of anti-Ro/SS-A and anti-La/SS-B in high values in both and FANA positivity in her mother (titer: 1/3200).

Fortunately, any heart block or other cardiologic abnormalities were not found by the pediatric cardiologist, and other laboratory evaluations were within the normal range.

These data confirmed the diagnosis of NLE without internal involvement for our patient.

Considering the fact that extensive capillary malformation was previously reported as a rare manifestation of NLE [3] and also normal ophthalmologic and neurologic evaluations in our patient, the diagnosis of SWS seemed to be unlikely and port-wine stain was assumed to be an uncommon feature of her NLE.

Surprisingly, she developed an episode of nonfebrile tonic-clonic seizure about one month later. Hence, she was admitted and received phenytoin, phenobarbital, and Tegretol. Brain CT scan revealed mild subdural hematoma and streaky calcification compatible with leptomeningeal

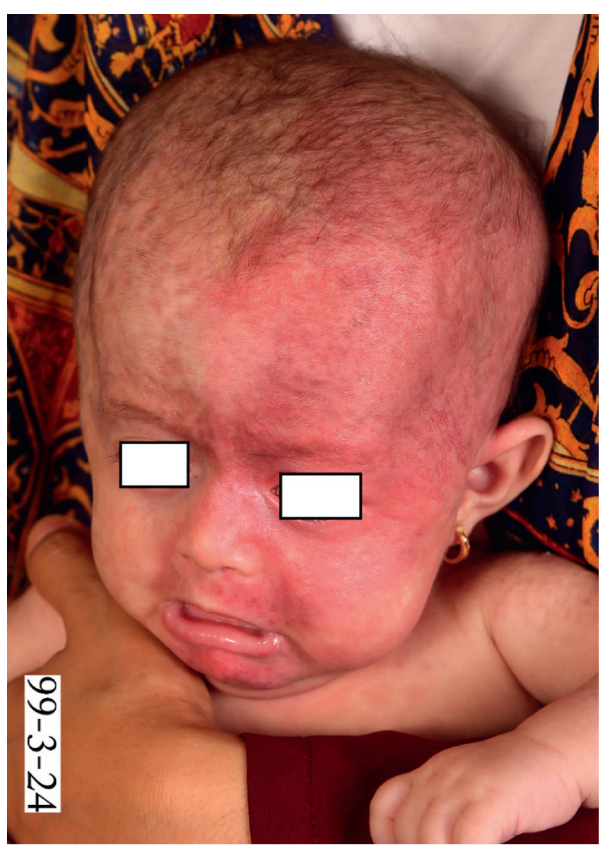

FIgURE 1: Extensive port-wine stain since birth.

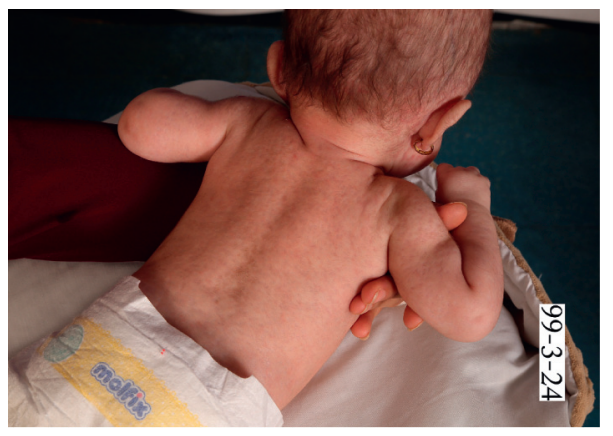

FIGURE 2: Multiple erythematous/hyperpigmented macules.

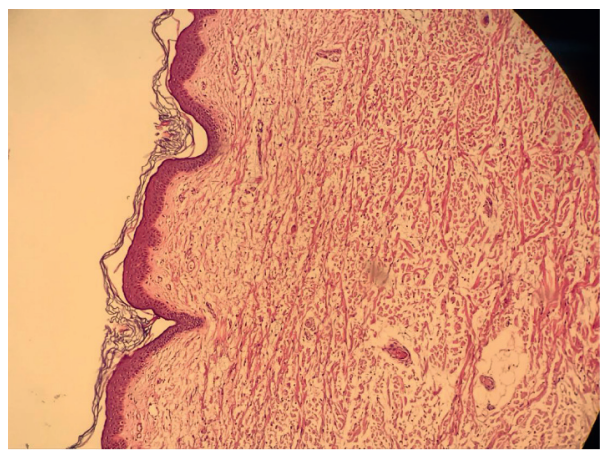

FIgURE 3: Dermal infiltration of spindle cells in a collagenous stroma containing abundant mucin intermixed with some mast cells.

angiomatosis which, in context of having an extensive portwine stain, returned us to the diagnosis of SWS. A secondary ophthalmologic consultation was requested in which glaucoma was noted in the left eye and the diagnosis was even more confirmed. 
Then, the patient was discharged on phenobarbital $15 \mathrm{mg} / \mathrm{BD}$ and syrup Tegretol $60 \mathrm{mg} / \mathrm{BD}$.

The patient remained seizure-free until the $10^{\text {th }}$ month of her life when the second episode of tonic-clonic seizure happened, and her medication was changed to phenobarbital, clonazepam, and aspirin. A brain MRI was performed which revealed leptomeningeal enhancement and hypotrophy in the left cerebral hemisphere indicative of SWS.

Now, the child is 15 months old. No seizure occurred within the last 5 months. Her port-wine stain considerably improved following 4 sessions of PDL (pulsed-dye laser) therapy and other cutaneous lesions getting better spontaneously (Figures 5 and 6).

\section{Discussion}

We reported a case of NLE and SWS where both of them are considered as rare conditions. To the best of our knowledge, the coexistence of these disorders has not been reported until now.

NLE is an uncommon disorder caused by transplacental transfer of maternal anti-Ro and anti-La autoantibodies. The most common manifestation of NLE is cutaneous lesions developed in $50 \%$ of cases, and they usually appear in the first months of life [5] but can be noted since birth in a minority of cases, such as ours.

In our patient, most of the lesions presented as atrophic and telangiectatic macules on the scalp and trunk which is more likely representative of occurrence of inflammatory phase during pregnancy. This might explain the absence of distinct inflammatory features of LE on histopathologic evaluation. In fact, the absence of typical annular lesions of NLE, as well as unspecific histopathologic findings in this case, indicates the importance of having clinical suspicion for reaching correct diagnosis.

It is worthy to note that the presence of abundant mucin in histopathologic assessment might be a feature of NLE lesions [5] and should not mislead us to more common differential diagnoses such as different types of mucinosis in case of clinical suspicion to LE.

Widespread cutaneous lesions of NLE at birth are very rare and might be considered as a proof for the hypothesis that UV radiation, despite previous reports [6], might not have an essential role in the expression of intranuclear antigen and developing clinical lesions.

Another interesting feature of our case was the fact that her mother initially seemed to be healthy, but a more detailed history revealed that she had arthralgia and hence was referred to the rheumatology department where it turned out that she had some criteria of SLE.

SWS is also a rare syndrome characterized with facial angioma reaching territory V1, ophthalmologic anomaly (mostly congenital glaucoma), and neurological malformation, such as leptomeningeal angioma might lead to seizure or neurological deficit [2]. SWS is classified into 3 types: type 1 (complete or classic), type 2 (bisymptomatic), and type 3 (rough form) [7].

Our case was initially presented by facial port-wine stain which could be considered as an uncommon cutaneous

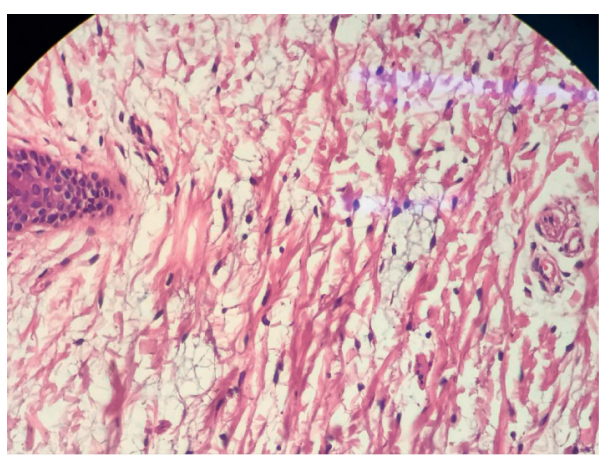

FIgURE 4: A magnified view of histopathological changes seen in the patient's tissue sample.

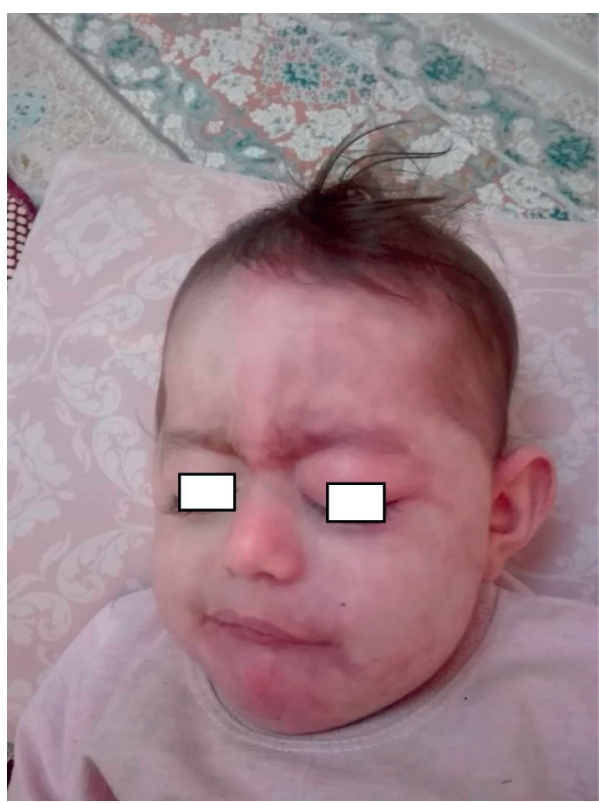

FIgURE 5: Remarkable resolution of port-wine stain following PDL therapy.

presentation of NLE considering normal first ophthalmologic and neurologic evaluation. However, occurrence of subsequent seizure episodes and detecting glaucoma on further assessments confirmed the diagnosis of SWS with complete manifestation (type 1).

As mentioned above, nothing abnormal was found on the first ophthalmologic assessment of our patient which might be due to developing glaucoma later, during the course of the disease.

Even though the most common form of ocular complication in SWS is congenital glaucoma, it can also develop during childhood or even later in life [8] which shows the significance of careful ophthalmic follow-up of these patients.

In conclusion, we report an extraordinary case of NLE not only for having lesions since birth and with lack of maternal history which posed a significant diagnostic challenge but also for simultaneously having another rare condition (SWS) which made it even more challenging to diagnosis. 


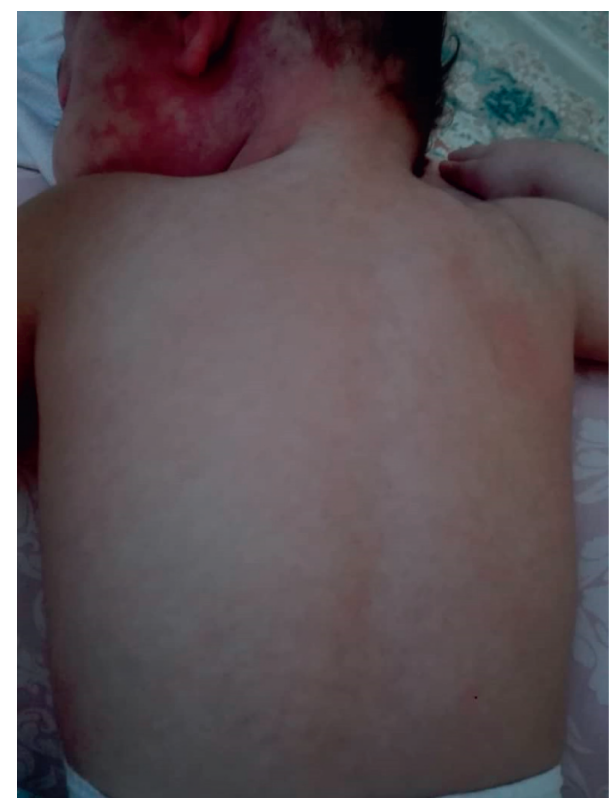

FIgURE 6: Spontaneous resolution of cutaneous lesions of NLE.

Making correct diagnosis in this case was important in several aspects: firstly, diagnosis of each of these disorders necessitates more systemic evaluations which led to detection of glaucoma in our case. Another important issue for patients with NLE is a relatively higher risk of developing other autoimmune disorders such as rheumatoid arthritis and Hashimoto thyroiditis during their childhood. Hence, they need long-term follow-up even though they experience spontaneous resolution of their extra cardiac manifestations [9].

Moreover, diagnosis of NLE in this case led to detection of SLE in her mother which was previously neglected. This also mandates strict follow-up of her mother during subsequent pregnancies due to higher risk of complete heart block in neonates which can be prevented by using IVIG and hydroxychloroquine early during pregnancy [10-14].

Therefore, careful examination and clinical suspicion besides appropriate paraclinical assessment are the key factors for correct diagnosis and management of patients.

\section{Data Availability}

Data are available on reasonable request to the corresponding authors.

\section{Consent}

The patient's parents provided written informed consent to publication of this case report and accompanying images.

\section{Conflicts of Interest}

The authors declare that they have no conflicts of interest regarding the publication of this article.

\section{Authors' Contributions}

Conception or design of the work was carried out by Z. N. Data were collected by Z. A. Data analysis and interpretation were performed by K. K. and A. G. The article was drafted by P. H. and Z. A. Critical revision of the article was conducted by Z. N. and A. G. Final approval of the version to be published was given by P. H. and Z. N.

\section{Acknowledgments}

This study was performed at the Autoimmune Bullous Diseases Research Center, Razi Hospital, Tehran, Iran.

\section{References}

[1] T. Chaikul and S. Chaithirayanon, "Unusual skin manifestations in neonatal lupus erythematosus," Medical Journal of the Medical Association of Thailand, vol. 98, no. Suppl 10, pp. S147-S149, 2015.

[2] I. Niang, K. M. Ndiaye, A. M. Diop et al., "Sturge Weber syndrome, when brain CT is enough for diagnosis: about a case," Pan African Medical Journal, vol. 36, p. 308, 2020.

[3] F. Vílchez-Márquez, A. Martín-Fuentes, R. Conejero et al., "Neonatal lupus erythematosus mimicking extensive capillary malformation," Pediatric Dermatology, vol. 30, no. 4, pp. 495-497, 2013.

[4] Y. Yu, L. Du, J. Pan, J. Zheng, A. Chen, and L. Chen, "A 10-year retrospective study of neonatal lupus erythematous in China," Asian Pacific Journal of Allergy and Immunology, vol. 34, no. 2, pp. 174-178, 2016.

[5] V. Vega, M. Duque, D. Don-Pedro, and S. Bhatta, "A neonate with annular cutaneous lesions," Cureus, vol. 12, no. 7, Article ID e9138, 2020.

[6] S. Admani and A. C. Krakowski, "Neonatal lupus erythematosus presenting as atypical targetoid-like lesions involving genitals and soles of feet following brief sun exposure," The Journal of clinical and aesthetic dermatology, vol. 6, no. 5, pp. 19-23, 2013.

[7] L. Siri, L. Giordano, P. Accorsi et al., "Clinical features of Sturge-Weber syndrome without facial nevus: five novel cases," European Journal of Paediatric Neurology, vol. 17, no. 1, pp. 91-96, 2013.

[8] P. C. Luna, "Transplacentally acquired dermatoses," Harper's Textbook of Pediatric Dermatology, vol. Volume 1, Wiley, Hoboken, NJ, USA, Fourth Edition, Chapter 8, , pp. 93-100, 2019.

[9] M. Silverstein and J. Salvin, "Ocular manifestations of SturgeWeber syndrome," Current Opinion in Ophthalmology, vol. 30, no. 5, pp. 301-305, 2019.

[10] A. Saxena, P. M. Izmirly, B. Mendez, J. P. Buyon, and D. M. Friedman, "Prevention and treatment in utero of autoimmune-associated congenital heart block," Cardiology in Review, vol. 22, no. 6, pp. 263-267, 2014.

[11] A. Brucato, R. Cimaz, R. Caporali, V. Ramoni, and J. Buyon, "Pregnancy outcomes in patients with autoimmune diseases and anti-Ro/SSA antibodies," Clinical Reviews in Allergy and Immunology, vol. 40, no. 1, pp. 27-41, 2011.

[12] P. M. Izmirly, M. Y. Kim, C. Llanos et al., "Evaluation of the risk of anti-SSA/Ro-SSB/La antibody-associated cardiac 
manifestations of neonatal lupus in fetuses of mothers with systemic lupus erythematosus exposed to hydroxychloroquine," Annals of the Rheumatic Diseases, vol. 69, no. 10, pp. 1827-1830, 2010.

[13] C. N. Pisoni, A. Brucato, A. Ruffatti et al., "Failure of intravenous immunoglobulin to prevent congenital heart block: findings of a multicenter, prospective, observational study," Arthritis \& Rheumatism, vol. 62, no. 4, pp. 1147-1152, 2010.

[14] D. M. Friedman, C. Llanos, P. M. Izmirly et al., "Evaluation of fetuses in a study of intravenous immunoglobulin as preventive therapy for congenital heart block: results of a multicenter, prospective, open-label clinical trial," Arthritis \& Rheumatism, vol. 62, no. 4, pp. 1138-1146, 2010. 